\title{
The generalized partial transposition criterion for separability of multipartite quantum states
}

\author{
Kai Chen and Ling-An Wu \\ Laboratory of Optical Physics, Institute of Physics, \\ Chinese Academy of Sciences, Beijing 100080, P.R. China,
}

\begin{abstract}
We present a generalized partial transposition separability criterion for the density matrix of a multipartite quantum system. This criterion comprises as special cases the famous Peres-Horodecki criterion and the recent realignment criterion in [O. Rudolph, quant-ph/0202121] and [K. Chen, L.A. Wu, quant-ph/0205017. It involves only straightforward matrix manipulations and is easy to apply. A quantitative measure of entanglement based on this criterion is also obtained.
\end{abstract}

PACS numbers: 03.67.-a, 03.65.Ud, 03.65.Ta

Keywords: Separability; Density matrix; Multipartite quantum system; Measure of entanglement

\section{INTRODUCTION}

Since the well-known papers of Einstein, Podolsky and Rosen [3], Schrödinger [4] and Bell [5], quantum entangled states have greatly enriched quantum mechanics and have recently found wide applications in the rapidly expanding field of quantum information processing. Quantum teleportation, quantum cryptography, quantum dense coding and parallel computation [6, [7, 8] have spurred a flurry of activity in the effort to fully exploit the potential of quantum entanglement. Although their applications have already been demonstrated experimentally, the physical character and mathematical structure of entangled states are only partially known, and a full comprehensive understanding is still a challenge for the theorists.

The most familiar entangled state is the singlet pure state of a pair of spin- $\frac{1}{2}$ particles given by Bohm [9]

$$
\psi_{s}=\frac{1}{\sqrt{2}}(|\uparrow\rangle|\downarrow\rangle-|\downarrow\rangle|\uparrow\rangle)
$$

which cannot be reduced or factorized to a direct product of the states for the two particles. Due to uncontrolled interactions with the environment, a practical composite system generally evolves to a mixed state. How do we know if a quantum state is entangled, and how entangled is it still after the intervention of noise?

To answer these questions we must have a physical acceptable definition of entangled states. From a physically meaningful and practical point of view, the state of a composite quantum system is called disentangled or separable if it can be prepared in a "local" or "classical" way. A separable multipartite system can be expressed as an ensemble realization of pure product states $\left|\psi_{i}\right\rangle_{A}\left|\phi_{i}\right\rangle_{B} \cdots\left|\varphi_{i}\right\rangle_{Z}$ occurring with a certain probability

\footnotetext{
*Electronic address: kchen@aphv.iphv.ac.cn

${ }^{\dagger}$ Electronic address: wula@aphy.iphy.ac.cn
}

$p_{i}$ :

$$
\rho_{A B \cdots Z}=\sum_{i} p_{i} \rho_{i}^{A} \otimes \rho_{i}^{B} \otimes \cdots \otimes \rho_{i}^{Z}
$$

where $\rho_{i}^{A}=\left|\psi_{i}\right\rangle_{A}\left\langle\psi_{i}\left|, \rho_{i}^{B}=\right| \phi_{i}\right\rangle_{B}\left\langle\phi_{i}\right|, \cdots, \rho_{i}^{Z}=$ $\left|\varphi_{i}\right\rangle_{Z}\left\langle\varphi_{i}\right|, \sum_{i} p_{i}=1$, and $\left|\psi_{i}\right\rangle_{A},\left|\phi_{i}\right\rangle_{B}, \cdots,\left|\varphi_{i}\right\rangle_{Z}$ are normalized pure states of subsystems $A, B, \cdots$, and $Z$, respectively 10. If no convex linear combination exists for a given $\rho_{A B} \cdots Z$, then the state is called "entangled" and includes quantum correlation.

For a pure state $\rho_{A B \cdots Z}$, it is trivial and straightforward to judge its separability:

A pure state $\rho_{A B \cdots Z}$ is separable if and only if

$$
\rho_{A B \cdots Z}=\rho_{A} \otimes \rho_{B} \otimes \cdots \otimes \rho_{Z},
$$

where $\rho_{A, B, \cdots, Z}$ are the reduced density matrices defined as $\rho_{A}=\operatorname{Tr}_{B, C, \cdots, Z}\left(\rho_{A B \cdots Z}\right), \rho_{B}=\operatorname{Tr}_{A, C, \cdots, Z}\left(\rho_{A B \cdots Z}\right)$, $\cdots, \rho_{Z}=\operatorname{Tr}_{A, B, \cdots, Y}\left(\rho_{A B \cdots Z}\right)$.

However, for a generic mixed state $\rho_{A B \cdots Z}$, finding a decomposition as in Eq. (2) or proving that it does not exist is a non-trivial task (we refer to recent reviews [11, 12, 13] and references therein). There has been considerable effort in recent years to analyze the separability and quantitative character of quantum entanglement. The Bell inequalities satisfied by a separable system give the first necessary condition for separability [5]. In 1996, Peres made an important step forward and showed that partial transpositions with respect to one and more subsystems of the density matrix for a separable state are positive,

$$
\rho^{T \mathcal{X}} \geq 0
$$

where $\mathcal{X} \subset\{A, B, \cdots, Z\}$. Thus the $\rho^{T \mathcal{X}}$ should have non-negative eigenvalues (this is known as the PPT criterion or Peres-Horodecki criterion) [14]. This was immediately shown by Horodecki et al 15 to be sufficient for bipartite systems of $2 \times 2$ and $2 \times 3$. Meanwhile, they also found a necessary and sufficient condition for separability by establishing a close connection between positive map theory and separability [15]. In view of the quantitative 
character for entanglement, Wootters succeeded in computing the "entanglement of formation" [16 and thus obtained a separability criterion for $2 \times 2$ mixtures [17. The "reduction criterion" proposed independently in 18] and 19] gives another necessary criterion which is equivalent to the PPT criterion for $2 \times n$ composite systems but is generally weaker. Pittenger et al gave also a sufficient criterion for separability connected with the Fourier representations of density matrices [20. Later, Nielsen et al 21] presented another necessary criterion called the $m a-$ jorization criterion: the decreasingly ordered vector of the eigenvalues for $\rho_{A B}$ is majorized by that of $\rho_{A}$ or $\rho_{B}$ alone for a separable state. A new method of constructing entanglement witnesses for detecting entanglement was given in 15] and 22, 23. . There are also some necessary and sufficient criteria of separability for low rank cases of the density matrix, as shown in [24, 25]. In addition, it was shown in [26] and 27 that a necessary and sufficient separability criterion is also equivalent to certain sets of equations.

However, despite these advances, a practical and convenient computable criterion for generic bipartite systems is mainly limited to the PPT, reduction and majorization criteria, as well as a recent extension of the PPT criterion based on semidefinite programs [28]. Very recently Rudolph [1] and the authors [2] proposed a new operational criterion for separability: the realignment criterion (named thus following the suggestion of 29] which is also called the computational cross norm criterion given in Ref. [1]). The criterion is very simple to apply and shows dramatic ability to detect most of the bound entangled states [2] and even genuinely tripartite entanglement 29]. This is a surprising new result, for the bound entangled states 30] are "weakly" inseparable and in the past it was very hard to establish with certainty their inseparability 19. Soon after, Horodecki et al showed that the PPT criterion and realignment criterion can be equivalent to any permutation of the indices of the density matrix [29].

In this paper we generalize our realignment criterion to multipartite quantum systems in arbitrary dimensions; then, from a different aspect, we give a systematic construction for the generalized partial transposition criterion. The constructions are given in Section II where the strong PPT and realignment criteria are shown as two special cases of the criterion. Only involving straightforward matrix manipulations, it is also very easy to apply. A quantitative measure of entanglement based on the criterion for detecting entanglement is also obtained in Section III. A brief summary and some discussions are given in the last section.

\section{THE CRITERIA FOR SEPARABILITY}

In this section we will give two criteria for separability of the density matrix. We first introduce the multipartite generalization of the realignment criterion, then present the generalized partial transposition criterion as a further generalization of the PPT and realignment criteria.

\section{A. Some notation}

The various matrix operations we shall use can be found in 31, 32], with the following notation:

Definition: For each $m \times n$ matrix $A=\left[a_{i j}\right]$, where $a_{i j}$ is the matrix entry of $A$, we define the vector vec $(A)$ as

$\operatorname{vec}(A)=\left[a_{11}, \cdots, a_{m 1}, a_{12}, \cdots, a_{m 2}, \cdots, a_{1 n}, \cdots, a_{m n}\right]^{T}$.

Let $Z$ be an $m \times m$ block matrix with block size $n \times n$. We define a "realignment" operation $\mathcal{R}$ to change $Z$ to a realigned matrix $\widetilde{Z}$ of size $m^{2} \times n^{2}$ that contains the same elements as $Z$ but in different positions as

$$
\mathcal{R}(Z) \equiv \widetilde{Z} \equiv\left[\begin{array}{c}
\operatorname{vec}\left(Z_{1,1}\right)^{T} \\
\vdots \\
\operatorname{vec}\left(Z_{m, 1}\right)^{T} \\
\vdots \\
\operatorname{vec}\left(Z_{1, m}\right)^{T} \\
\vdots \\
\operatorname{vec}\left(Z_{m, m}\right)^{T}
\end{array}\right]
$$

For example, a $2 \times 2$ bipartite density matrix $\rho$ can be transformed as:

$$
\begin{aligned}
\rho & =\left(\begin{array}{ll|ll}
\rho_{11} & \rho_{12} & \rho_{13} & \rho_{14} \\
\rho_{21} & \rho_{22} & \rho_{23} & \rho_{24} \\
\hline \rho_{31} & \rho_{32} & \rho_{33} & \rho_{34} \\
\rho_{41} & \rho_{42} & \rho_{43} & \rho_{44}
\end{array}\right) \\
& \longrightarrow \mathcal{R}(\rho)=\left(\begin{array}{llll}
\rho_{11} & \rho_{21} & \rho_{12} & \rho_{22} \\
\hline \rho_{31} & \rho_{41} & \rho_{32} & \rho_{42} \\
\hline \rho_{13} & \rho_{23} & \rho_{14} & \rho_{24} \\
\hline \rho_{33} & \rho_{43} & \rho_{34} & \rho_{44}
\end{array}\right) .
\end{aligned}
$$

\section{B. The realignment criterion}

Motivated by the Kronecker product approximation technique for a matrix [33, 34], we developed a very simple method to obtain the realignment criterion in [2].

The realignment criterion: If an $m \times n$ bipartite density matrix $\rho_{A B}$ is separable, then for the $m^{2} \times n^{2}$ matrix $\mathcal{R}\left(\rho_{A B}\right)$ the trace norm $\left\|\mathcal{R}\left(\rho_{A B}\right)\right\| \equiv \sum_{i=1}^{q} \sigma_{i}\left(\mathcal{R}\left(\rho_{A B}\right)\right)$, which is the sum of all the singular values of $\mathcal{R}\left(\rho_{A B}\right)$, should be $\leq 1$, or equivalently $\log \left\|\mathcal{R}\left(\rho_{A B}\right)\right\| \leq 0$ where $q=\min \left(m^{2}, n^{2}\right)$.

This criterion is strong enough to detect most of the bound entangled states in the literature, as shown in [2].

For multipartite systems, we have a natural generalization that was also partially introduced in [29]: 
The multipartite realignment criterion: If an $n$-partite density matrix $\rho$ is separable, then

$$
\left\|\left(\mathcal{R}_{(k)} \otimes I_{(n-k)}\right) \rho\right\| \leq 1, \quad k=2,3, \cdots, n
$$

where the subscript indices mean that we act by $\mathcal{R}_{(k)}$ on $k$ chosen subsystems, while leaving untouched the remaining $n-k$ subsystems.

Here $\mathcal{R}_{(k)}$ can be a realigned matrix according to all the possible bipartite cuts for the $k$ subsystems, in addition to all their combinations. It is surprisingly strong enough to detect the genuinely tripartite bound entangled state [29] which is bi-separable with respect to any bipartite cuts for the 3 subsystems. The operation $\left(\mathcal{R}_{(2)} \otimes I_{(n-2)}\right)$ is also shown to be equivalent to certain permutations of the indices of the density matrix [29].

\section{The generalized partial transposition criterion}

We will now derive the main result of this paper: a generalized partial transposition criterion for separability of multipartite quantum systems in arbitrary dimensions.

\section{The main theorem}

Since the density matrix $\rho_{p}$ for a $d$-dimensional pure separable state is a self-adjoint Hermitian $d \times d$ matrix with only one eigenvalue 1 , it is evident that $\rho_{p}$ is of rank 1 and we have naturally

$$
\rho_{p}=u \otimes u^{\dagger}=u^{\dagger} \otimes u,
$$

where $u$ is a $d \times 1$ column vector satisfying $u^{\dagger} u \equiv 1$. This is possible since $u$ can be chosen to be the normalized eigenvector of $\rho_{p}$. For the convenience of later use we define two new operators $\mathcal{T}_{r}, \mathcal{T}_{c}$ and their multiplication:

$$
\begin{aligned}
\mathcal{T}_{r} & : A \longrightarrow \text { row transposition of } A \\
& \Longleftrightarrow A \longrightarrow(\operatorname{vec}(A))^{t}, \\
\mathcal{T}_{c} & : A \longrightarrow \operatorname{column} \text { transposition of } A \\
& \Longleftrightarrow A \longrightarrow \operatorname{vec}(A),
\end{aligned}
$$$$
\mathcal{T}_{c} \mathcal{T}_{r} \text { or } \mathcal{T}_{r} \mathcal{T}_{c}: A \longrightarrow A^{t},
$$

where $t$ represents standard transposition operation. For example, a $2 \times 2$ matrix $A$ can be transformed to

$$
\begin{aligned}
& A=\left(\begin{array}{ll}
a_{11} & a_{12} \\
a_{21} & a_{22}
\end{array}\right)
\end{aligned}
$$

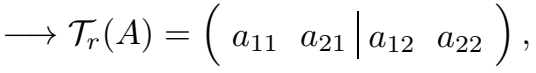

$$
\begin{aligned}
& \longrightarrow \mathcal{T}_{c}(A)=\left(\begin{array}{c}
a_{11} \\
\frac{a_{21}}{a_{12}} \\
a_{22}
\end{array}\right) \text {. }
\end{aligned}
$$

As for a generic density matrix we have $\rho=$ $\sum_{i, j} \rho_{i j}|i\rangle\left\langle j\left|=\sum_{i, j} \rho_{i j}\right| i\right\rangle \otimes\langle j|=\sum_{i, j} \rho_{i j}\langle j|\otimes| i\rangle$ where $|i\rangle,|j\rangle$ are suitably selected normalized orthogonal bases and $\langle i|,\langle j|$ are the corresponding transpositions, respectively (here, for simplicity of notation, $\langle i|,\langle j|$ are not regarded as the corresponding conjugate transpositions). Thus the operations of $\mathcal{T}_{r}$ and $\mathcal{T}_{c}$ can be realized conveniently:

$$
\begin{aligned}
& \rho \stackrel{\mathcal{T}_{r}}{\longrightarrow} \sum_{i, j} \rho_{i j}\langle j| \otimes\left\langle i\left|\stackrel{\mathcal{T}_{c}}{\longrightarrow} \sum_{i, j} \rho_{i j}\right| j\right\rangle \otimes\langle i|=\rho^{t}, \\
& \rho \stackrel{\mathcal{T}_{c}}{\longrightarrow} \sum_{i, j} \rho_{i j}|j\rangle \otimes|i\rangle \stackrel{\mathcal{T}_{r}}{\longrightarrow} \sum_{i, j} \rho_{i j}|j\rangle \otimes\langle i|=\rho^{t} .
\end{aligned}
$$

Now we arrive at the following separability criterion for a multipartite system:

Theorem 1: If an $n$-partite $d_{1} \times d_{2} \times \cdots \times d_{n}$ density matrix $\rho$ is separable, then the generalized partial transpositions of $\rho$ satisfy

$$
\left\|\rho^{\mathcal{T}_{\mathcal{Y}}}\right\| \leq 1, \quad \forall \mathcal{Y} \subset\{\underbrace{r_{A}, c_{A}, r_{B}, c_{B}, \cdots, r_{Z}, c_{Z}}_{2 n}\}
$$

where $\mathcal{T}_{r_{k}}$ or $\mathcal{T}_{c_{k}}(k=A, B, \cdots, Z)$ means transposition with respect to the row or column for the kth subsystem. The superscript indices $\mathcal{T} \mathcal{Y}$ represent partial transpositions of every element of set $\mathcal{Y}$ on chosen subsystems, while leaving untouched the remaining subsystems.

Proof: Applying Eq. (2) for separable states and the property Eq. (8) for pure states, we have

$$
\rho=\sum_{i} p_{i} u_{i}^{A} \otimes u_{i}^{A \dagger} \otimes u_{i}^{B} \otimes u_{i}^{B \dagger} \otimes \cdots \otimes u_{i}^{Z} \otimes u_{i}^{Z \dagger} .
$$

The transformation of $\rho^{\mathcal{T} y}$ is to make partial transpositions of the rows or columns corresponding to some subsystems. Without loss of generality we suppose that we only make a row transposition to the $A$ subsystem:

$\rho^{\mathcal{T}_{\left\{r_{A}\right\}}}=\sum_{i} p_{i}\left(u_{i}^{A}\right)^{\dagger} \otimes\left(u_{i}^{A}\right)^{t} \otimes u_{i}^{B} \otimes u_{i}^{B \dagger} \otimes \cdots \otimes u_{i}^{Z} \otimes u_{i}^{Z \dagger}$.

We shall need the following property (see Chapters 3,4 of [32]) of the Kronecker product of the $m \times m$ matrix $A$ and the $n \times n$ matrix $B$ (Property 1), as well as that of the trace norm of two matrices $A$ and $B$ of the same size (Property 2):

Property 1: the non-zero singular values of $A \otimes B$ are the positive numbers $\left\{\sigma_{i}(A) \sigma_{j}(B)\right\}$ where $\sigma_{i}(A)$ and $\sigma_{j}(B)$ are non zero singular values of $A$ and $B$ arranged in decreasing order, respectively,

Property 2: $\|A+B\| \leq\|A\|+\|B\|$, 
so that

$$
\begin{aligned}
& \left\|\rho^{\mathcal{T}_{\left\{r_{A}\right\}}}\right\| \\
& \leq \sum_{i} p_{i}\left\|\left(u_{i}^{A}\right)^{\dagger} \otimes\left(u_{i}^{A}\right)^{t} \otimes u_{i}^{B} \otimes u_{i}^{B \dagger} \otimes \cdots \otimes u_{i}^{Z} \otimes u_{i}^{Z \dagger}\right\| .
\end{aligned}
$$

It is straightforward that $u_{i}^{A},\left(u_{i}^{A}\right)^{\dagger},\left(u_{i}^{A}\right)^{t}$ and $\left(u_{i}^{A}\right)^{*}$ have only one singular value 1 , respectively, due to the normalization condition $u_{i}^{A^{\dagger}} u_{i}^{A}=1$. The same holds for the case of $A$ replaced with $B, C, \cdots$, or $Z$. Thus we obtain $\left\|\left(u_{i}^{A}\right)^{\dagger} \otimes\left(u_{i}^{A}\right)^{t} \otimes u_{i}^{B} \otimes u_{i}^{B \dagger} \otimes \cdots \otimes u_{i}^{Z} \otimes u_{i}^{Z \dagger}\right\|=1$ by applying Property 1 . Therefore it is evident that

$$
\left\|\rho^{\mathcal{T}_{\left\{r_{A}\right\}}}\right\| \leq \sum_{i} p_{i}=1
$$

When applying column transposition, the standard transposition with respect to one subsystem or even a combination of transpositions to a subset $\mathcal{Y}$ of $\left\{r_{A}, c_{A}, r_{B}, c_{B}, \cdots, r_{Z}, c_{Z}\right\}$, we follow a similar procedure and obtain the same conclusion as the abovederived. Thus we obtain the final result of Eq. (14).

\section{Relationship with other necessary criteria}

We shall now show that Theorem 1 actually encompasses previous strong computational criteria for separability.

a. The PPT criterion For partial transposition with respect to one or more subsystems of a separable state, we have

$$
\rho^{T_{\mathcal{X}}} \geq 0
$$

and $\left\|\rho^{T_{\mathcal{X}}}\right\|=\operatorname{Tr}\left(\rho^{T_{\mathcal{X}}}\right)=1$ due to the Hermitian property of $\rho^{T_{\mathcal{X}}}$. It is obvious for any $\mathcal{X} \subset\{A, B, \cdots, Z\}$ that there is a $\mathcal{Y}$ in the generalized partial transposition criterion satisfying

$$
\left\|\rho^{\mathcal{T}_{\mathcal{Y}}}\right\|=1
$$

We only need to substitute $\mathcal{X}$ with its pairwise correspondence to $\mathcal{Y} \subset\left\{r_{A}, c_{A}, r_{B}, c_{B}, \cdots, r_{Z}, c_{Z}\right\}$. For example,

$$
\begin{aligned}
\mathcal{X} & =\{B, C\} \\
& \mathbb{} \\
\mathcal{Y} & =\left\{r_{B}, c_{B}, r_{C}, c_{C}\right\} .
\end{aligned}
$$

b. The multipartite realignment criterion From the property given in Ref. [34], we have a Kronecker product decomposition for a density matrix of $\rho=\sum_{i} \alpha_{i}^{A} \otimes \beta_{i}^{B}$ where $\alpha_{i}^{A}, \beta_{i}^{B}$ are, in general, not density matrices. Thus one finds

$$
\mathcal{R}(\rho)=\sum_{i} \operatorname{vec}\left(\alpha_{i}^{A}\right) \operatorname{vec}\left(\beta_{i}^{B}\right)^{T}
$$

it is easy to find that the above operation $\mathcal{R}(\rho)$ corresponds to $\mathcal{Y}=\left\{c_{A}, r_{B}\right\}$ from the properties of Eq. (9) and $(10)$. For the generic realignment operation $\left(\mathcal{R}_{(k)} \otimes\right.$ $\left.I_{(n-k)}\right)$, we will have a similar correspondence so that Theorem 1 also includes the multipartite realignment criterion of Eq. (7) as a special case.

c. The Horodecki indices permutation criterion As for the criterion proposed in 29], Horodecki et al show that the PPT criterion and the realignment criterion are equivalent to certain permutations of density matrix indices up to some unitary matrix which keeps the trace norm invariant. Moreover, it is obvious that the realignment criterion is identical to the operation of $\mathcal{R}_{(2)} \otimes I_{(n-2)}$ in the language of generic realignment, so it is also a special case of Theorem 1 according to (II C 2 b).

We can see that the generalized partial transposition criterion is a powerful computational criterion since it includes as special cases the strong PPT and the generic realignment criterion. This gives us a whole framework to detect entanglement with great convenience of manipulation. In addition, it also contains some new criteria for recognizing entanglement. For instance, making only the row transpositions or column transpositions of various subsystems is different from the PPT and realignment criteria. However, despite these virtues, it is still not sufficient for detecting all the entangled states. For example, it still fails to recognize the $2 \times 4$ Horodecki bound entangled state [30] by direct computation.

\section{ENTANGLEMENT MEASURE INDUCED BY THE SEPARABILITY CRITERION}

With the power of our new criterion, we expect that it should be able to give some information on the degree of entanglement. Among the quantitative measures, the entanglement of formation $E_{F}(\rho)$ 16 and distillable entanglement $E_{D}(\rho)$ 16] are two of the most meaningful ones. The former quantifies the asymptotic pure-state entanglement required to create $\rho$ while the latter quantifies which can be extracted from $\rho$, by means of local operations and classical communication (LOCC). But, in practice it is very difficult to compute the two measures for a generic quantum state. There is only one exception for the success of computation of $E_{F}(\rho)$ for two-qubits [17.

Recently, Vidal and Werner showed that the negativity is an entanglement monotone [35] and therefore a good entanglement measure [36]. The negativity is defined as

$$
\mathcal{N}(\rho) \equiv \frac{\left\|\rho^{T_{A}}\right\|-1}{2}
$$

Following this idea we introduce a computational measure based on our criterion as

$E(\rho) \equiv \sup \frac{\left\|\rho^{\mathcal{T}_{\mathcal{Y}}}\right\|-1}{2}, \quad \mathcal{Y} \subset\left\{r_{A}, c_{A}, r_{B}, c_{B}, \cdots, r_{Z}, c_{Z}\right\}$ 
For separable states, $\left\|\rho^{\mathcal{T}_{\mathcal{Y}}}\right\| \leq 1$ due to Theorem 1 . Whenever $\mathcal{Y}$ corresponds to a standard partial transposition of one or more of the subsystems, we have $\left\|\rho^{\mathcal{T}_{\mathcal{Y}}}\right\|=1$ due to the positivity of $\rho^{\mathcal{T}_{\mathcal{y}}}$. Thus we have $E(\rho)=0$ for all the separable states. On the other hand, $E(\rho)$ is convex due to the convexity of the trace norm. Furthermore, we have the following result:

Theorem 2: Applying a local unitary transformation leaves $E(\rho)$ invariant, i.e.

$$
E\left(\rho^{\prime}\right)=E(\rho)
$$

where $\rho^{\prime}=\left(U_{A} \otimes U_{B} \otimes \cdots \otimes U_{Z}\right) \rho\left(U_{A}^{\dagger} \otimes U_{B}^{\dagger} \otimes \cdots \otimes U_{Z}^{\dagger}\right)$ and $U_{A}, U_{B}, \cdots, U_{Z}$ are unitary operators acting on $A$, $B, \cdots, Z$ subsystems, respectively.

Proof: If $\left\|\rho^{\mathcal{T}_{\mathcal{Y}}}\right\|=\left\|\left(\rho^{\prime}\right)^{T_{\mathcal{Y}}}\right\|$ for $\forall \mathcal{Y}$, we certainly have Eq. (24). Consider one term of $\rho^{\prime}$ :

$$
\begin{aligned}
\rho_{i}^{\prime} & =\left(U_{A} \otimes U_{B} \otimes \cdots \otimes U_{Z}\right)\left(\alpha_{i}^{A} \otimes \beta_{i}^{B} \otimes \cdots \otimes \eta_{i}^{Z}\right) \\
& \times\left(U_{A}^{\dagger} \otimes U_{B}^{\dagger} \otimes \cdots \otimes U_{Z}^{\dagger}\right) \\
& =U_{A} \alpha_{i}^{A} U_{A}^{\dagger} \otimes U_{B} \beta_{i}^{B} U_{B}^{\dagger} \otimes \cdots \otimes U_{Z} \eta_{i}^{Z} U_{Z}^{\dagger},
\end{aligned}
$$

where we suppose $\rho=\sum_{i} \alpha_{i}^{A} \otimes \beta_{i}^{B} \otimes \cdots \otimes \eta_{i}^{Z}$ which can be obtained according to [37] or can be done by applying repeatedly the Kronecker product decomposition for a bipartite-cut of a matrix 33, 34]. It should be remarked that $\alpha_{i}^{A}, \beta_{i}^{B}, \cdots, \eta_{i}^{Z}$ are, in general, not density matrices. Without loss of generality, we can just perform a row transposition on the $A$ subsystem, thus

$\left(\rho_{i}^{\prime}\right)^{\mathcal{T}_{\left\{r_{A}\right\}}}=\left(\operatorname{vec}\left(U_{A} \alpha_{i}^{A} U_{A}^{\dagger}\right)\right)^{t} \otimes U_{B} \beta_{i}^{B} U_{B}^{\dagger} \otimes \cdots \otimes U_{Z} \eta_{i}^{Z} U_{Z}^{\dagger}$.

Applying the property

$$
\operatorname{vec}(X Y Z)=\left(Z^{T} \otimes X\right) \operatorname{vec}(Y), \quad(\text { see 32) }
$$

we arrive at

$$
\begin{aligned}
\left(\rho_{i}^{\prime}\right)^{\mathcal{T}_{\left\{r_{A}\right\}}} & =\left(\left(U_{A}^{*} \otimes U_{A}\right) \operatorname{vec}\left(\alpha_{i}^{A}\right)\right)^{t} \otimes U_{B} \beta_{i}^{B} U_{B}^{\dagger} \\
& \otimes \cdots \otimes U_{Z} \eta_{i}^{Z} U_{Z}^{\dagger} \\
& =\left(I d \otimes U_{B} \otimes \cdots \otimes U_{Z}\right) \\
& \times\left(\left(\operatorname{vec}\left(\alpha_{i}^{A}\right)\right)^{t} \otimes \beta_{i}^{B} \otimes \cdots \otimes \eta_{i}^{Z}\right) \\
& \times\left(U_{A}^{\dagger} \otimes U_{A}^{t} \otimes U_{B}^{\dagger} \otimes \cdots \otimes U_{Z}^{\dagger}\right)
\end{aligned}
$$

Since a local unitary transformation acts as a whole factor on $\rho$, we have

$$
\begin{aligned}
\|\left(\rho^{\prime}\right)^{\mathcal{T}_{\left\{r_{A}\right\}} \|} & =\|\left(I d \otimes U_{B} \otimes \cdots \otimes U_{Z}\right) \\
& \times \sum_{i}\left(\left(\operatorname{vec}\left(\alpha_{i}^{A}\right)\right)^{t} \otimes \beta_{i}^{B} \otimes \cdots \otimes \eta_{i}^{Z}\right) \\
& \times\left(U_{A}^{\dagger} \otimes U_{A}^{t} \otimes U_{B}^{\dagger} \otimes \cdots \otimes U_{Z}^{\dagger}\right) \| \\
& =\left\|\sum_{i}\left(\left(\operatorname{vec}\left(\alpha_{i}^{A}\right)\right)^{t} \otimes \beta_{i}^{B} \otimes \cdots \otimes \eta_{i}^{Z}\right)\right\| \\
& =\|(\rho)^{\mathcal{T}_{\left\{r_{A}\right\}} \| .}
\end{aligned}
$$

Here we have used the unitarily invariant property of the trace norm.

The same procedure can be used to perform column transposition, partial transposition of some subsystems, and any combinations of these transpositions. The proof is thus completed.

Since the negativity gives an upper bound for the distillable entanglement, we also obtain an upper bound $E(\rho)$ for the distillable entanglement $E_{D}(\rho)$, because $E(\rho)$ takes the maximum for all the possible $\mathcal{Y}$ transpositions which naturally contain the partial transposition operations.

\section{CONCLUSION}

Summarizing, we have presented a computational necessary criterion for separability of multipartite quantum systems in arbitrary dimensions which we call a "generalized partial transposition criterion". This criterion unifies the previous known PPT criterion, the recently found realignment criterion and the permutation criterion in a single concise framework. It provides a very powerful necessary condition for separability and is quite easy to apply. A quantitative measure of entanglement based on the criterion is also obtained. Moreover, the measure gives an upper bound for distillable entanglement.

Comparing with previous works, we have significantly expanded our ability to distinguish directly the entanglement and separability of any multipartite quantum state in arbitrary dimensions. We expect that our method can shed some light on the final solution of the separability problem and provide a more suitable better measure for the degree of entanglement.

\section{ACKNOWLEDGMENTS}

We would like to thank Dr. H. Fan and Dr. L. Yang for valuable discussions. K.C. is grateful to encouragement from Prof. Guozhen Yang. This work was supported by the National "973" Program for Fundamental Research, the Knowledge Innovation Program of the Chinese Academy of Sciences and the National Natural Science Foundation of China. 
[1] O. Rudolph, quant-ph/0202121.

[2] K. Chen and L.A. Wu, quant-ph/0205017.

[3] A. Einstein, B. Podolsky and N. Rosen, Phys. Rev. 47 (1935) 777.

[4] E. Schrödinger, Natürwissenschaften 23 (1935) 807.

[5] J.S. Bell, Physics (N.Y.) 1 (1964) 195.

[6] J. Preskill, The Theory of Quantum Information and Quantum Computation, California Inst. of Tech., Pasadena, CA, 2000, http://www.theory.caltech.edu/people/preskill/ph229/.

[7] M.A. Nielsen and I.L. Chuang, Quantum Computation and Quantum Information, Cambridge University Press, Cambridge, 2000.

[8] D. Bouwmeester, A. Ekert and A. Zeilinger (Eds.), The Physics of Quantum Information: Quantum Cryptography, Quantum Teleportation and Quantum Computation, Springer, New York, 2000.

[9] D. Bohm, Phys. Rev. 85 (1952) 166.

[10] R.F. Werner, Phys. Rev. A 40 (1989) 4277.

[11] M. Horodecki, P. Horodecki and R. Horodecki, Springer Tracts in Mod. Phy. 173 (2001) 151.

[12] M. Lewenstein, D. Bruss, J.I. Cirac, B. Kraus, M. Kus, J. Samsonowicz, A. Sanpera and R. Tarrach, J. Mod. Opt. 47 (2000) 2841.

[13] B.M. Terhal, Theor. Comput. Sci. 287 (2002) 313.

[14] A. Peres, Phys. Rev. Lett. 77 (1996) 1413.

[15] M. Horodecki, P. Horodecki and R. Horodecki, Phys. Lett. A 223 (1996) 1.

[16] C.H. Bennett, D.P. DiVincenzo, J.A. Smolin and W.K. Wootters, Phys. Rev. A 54 (1996) 3824.

[17] W.K. Wootters, Phys. Rev. Lett. 80 (1998) 2245.

[18] M. Horodecki and P. Horodecki, Phys. Rev. A 59 (1999) 4206.

[19] N.J. Cerf, C. Adami and R.M. Gingrich, Phys. Rev. A 60 (1999) 898.

[20] A.O. Pittenger and M.H. Rubin, Phys. Rev. A 62 (2000)
032313.

[21] M.A. Nielsen and J. Kempe, Phys. Rev. Lett. 86 (2001) 5184.

[22] B. Terhal, Phys. Lett. A 271 (2000) 319.

[23] M. Lewenstein, B. Kraus, J.I. Cirac and P. Horodecki, Phys. Rev. A 62 (2000) 052310.

[24] P. Horodecki, M. Lewenstein, G. Vidal and I. Cirac, Phys. Rev. A 62 (2000) 032310.

[25] S. Albeverio, S.M. Fei and D. Goswami, Phys. Lett. A 286 (2001) 91.

[26] S.J. Wu, X.M. Chen and Y.D. Zhang, Phys. Lett. A 275 (2000) 244.

[27] P.X. Chen, L.M. Liang, C.Z. Li and M.Q. Huang, Phys. Rev. A 63 (2001) 052306.

[28] A.C. Doherty, P.A. Parrilo and F.M. Spedalieri, Phys. Rev. Lett. 88 (2002) 187904.

[29] M. Horodecki, P. Horodecki and R. Horodecki, quant$\mathrm{ph} / 0206008$.

[30] P. Horodecki, Phys. Lett. A 232 (1997) 333.

[31] R.A. Horn and C.R. Johnson, Matrix Analysis, Cambridge University Press, New York, 1985.

[32] R.A. Horn and C.R. Johnson, Topics in Matrix Analysis, Cambridge University Press, New York, 1991.

[33] C.F. Van Loan and N.P. Pitsianis, in: Linear Algebra for Large Scale and Real Time Applications, M.S. Moonen and G.H. Golub (Eds.), Kluwer Publications, 1993, pp. 293-314.

[34] N.P. Pitsianis, Ph.D. thesis, The Kronecker Product in Approximation and Fast Transform Generation Cornell University, New York, 1997.

[35] G. Vidal, J. Mod. Opt. 47 (2000) 355.

[36] G. Vidal and R.F. Werner, Phys. Rev. A 65 (2002) 032314.

[37] L. De Lathauwer, B. De Moor and J. Vandewalle, SIAM J. Matrix. Anal. Appl. 21 (2000) 1253. 